\title{
The Fuzzy Lattice of Ideals and Filters of an Almost Distributive Fuzzy Lattice
}

\author{
Berhanu Assaye Alaba and Bekalu Tarekegn Bitew
}

\begin{abstract}
In this paper, the concept of fuzzy lattice is discussed. It is proved that a fuzzy poset $\left(I_{A}(L), B\right)$ and $\left(F_{A}(L), B\right)$ forms a fuzzy lattice, where $I_{A}(L)$ and $F_{A}(L)$ are the set containing all ideals, and the set containing all filters of an Almost Distributive Fuzzy Lattice(ADFL) respectively. In addition we proved that, a fuzzy poset $\left(P I_{A}(L), B\right)$ and $\left(P F_{A}(L), B\right)$ forms fuzzy distributive lattice, where $P I_{A}$ and $P F_{A}(L)$ denotes the set containing all principal ideals and the set containing all principal filters of an ADFL. Finally, it is proved that for any ideal $I$ and filter $\mathbf{F}$ of an ADFL, $I_{A}^{i}=\left\{(i]_{A}: i \in I\right\}$ and $F_{A}^{f}=\left\{[f)_{A}: f \in F\right\}$ are ideals of a fuzzy distributive lattice $\left(P I_{A}(L), B\right)$ and $\left(P F_{A}(L), B\right)$ respectively, and $F_{A}^{i}=\left\{(f]_{A}: f \in F\right\}$ and $I_{A}^{f}=\left\{[i)_{A}: i \in I\right\}$ are filters of a distributive fuzzy lattice $\left(P I_{A}(L), B\right)$ and $\left(P F_{A}(L), B\right)$ respectively.
\end{abstract}

Index Terms-Almost distributive fuzzy lattice, filters, fuzzy lattice, ideals.

\section{INTRODUCTION}

$\mathbf{T}$ HE axiomatization of Boole's two valued propositional calculus led to the concept of Boolean Algebra and the class of Boolean Algebras(Ring). This includes the ring theoretic generalizations and the lattice theoretic generalizations like Heyting Algebras and distributive lattice. U.M.Samy and G.C.Rao [1] introduced the concepts of an ADL as a common abstraction of distributive lattice.

On the other hand, Zadeh [2] was the first mathematician to introduced the concepts of fuzzy and, to define and study fuzzy relations, Sanchez [3], Goguen [4] adapted this concept. The notion of partial order and lattice order goes back to 19 th century investigations in logic. The concepts of fuzzy sublattices and fuzzy ideals of a lattice was introduced by Yuan and Wu [5]. Fuzzy lattice was defined as a fuzzy algebra by Ajmal and Thomas [6] and they characterized fuzzy sublattices for a first time. In 2009, fuzzy partial order relation was characterized interms of its level set by Chon [7]. Chon in the same paper defined a fuzzy lattice as a fuzzy relation, developed basic properties and characterized a fuzzy lattice by its level set.

As a continuation of these studies, in 2016 Berhanu [8] define an Almost Distributive Fuzzy Lattice as a generalization of Distributive Fuzzy Lattice and fuzzyfy some properties of the classical Almost Distributive Lattice using the fuzzy partial order relation and fuzzy lattice defined by Chon [7]. In the same year Berhanu and Bekalu [9], introduced the concepts of ideals and filters of an Almost Distributive fuzzy lattice. In

Manuscript received November 27, 2016; accepted February 10, 2017.

The authors are with the Department of Mathematics, College of Science, Bahir Dar University, Bahir Dar, Ethiopia. Emails: \{berhanu_ assaye, t. bekalu\}@yahoo.com addition, Berhanu and Bekalu [10] in the same year, introduced the concepts of principal ideals and filters of an ADFL as a continuation of [9].

Again, as a continuation of Berhanu and Bekalu's [9] and [10] work, in this paper we introduce the concepts of fuzzy lattices by defining a fuzzy relation on the set containing all ideals of a given Almost Distributive Fuzzy Lattice, and on the set containing all filters of a given Almost Distributive Fuzzy Lattice. In addition, we proved that a fuzzy poset $\left(P I_{A}(L), B\right)$ and $\left(P F_{A}(L), B\right)$ forms a distributive fuzzy lattice, where $P I_{A}(L)$ is the set containing all principal ideals, and $P F_{A}(L)$ denotes the set containing all filters of a given ADFL L. Moreover in this paper, we introduce ideals and filters of the two distributive fuzzy lattice $\left(P I_{A}(L), B\right)$ and $\left(P F_{A}(L), B\right)$ induced by arbitrary ideal $I$ and filter $F$ of an ADFL L.

In this work, we will use Fuzzy Lattice defined by Chon [7], and an Almost Distributive Fuzzy Lattice introduced by Berhanu [8].

\section{Preliminaries}

In this section we recall some definitions, basic and important results from [9],[10],[8], and [7] that will be required in this paper.

In this paper, an Almost Distributive Fuzzy Lattice $(R, A)$ is denoted by $\mathrm{L}$, and $\mathrm{R}$ represents a non empty set such that $\mathrm{L}$ $=(\mathrm{R}, \mathrm{A})$ forms an Almost Distributive fuzzy lattice $(\mathrm{ADFL})$.

Definition 1 ([7]): Let $\mathrm{X}$ be a set. A function $A: X \times X \longrightarrow$ $[0,1]$ is called a fuzzy relation in $\mathrm{X}$. The fuzzy relation $\mathrm{A}$ in $\mathrm{X}$ is reflexive if and only if $A(x, x)=1$ for all $x \in X, A$ is transitive if and only if $A(x, z) \geq \sup _{y \in X} \min (A(x, y), A(y, z))$, and $\mathrm{A}$ is antisymmetric if and only if $A(x, y)>0$ and $A(y, x)>0$ implies $x=y$. A fuzzy relation $\mathrm{A}$ is fuzzy partial order relation if A is reflexive, antisymmetric and transitive. A fuzzy partial order relation A is a fuzzy total order relation if and only if $A(x, y)>0$ or $A(y, x)>0$ for all $x, y \in R$.If $\mathrm{A}$ is a fuzzy partial order relation in a set $\mathrm{X}$, then $(\mathrm{X}, \mathrm{A})$ is called a fuzzy partially ordered set or a fuzzy poset. If $\mathrm{B}$ is a fuzzy total order relation in a set $\mathrm{X}$, then $(X, B)$ is called a fuzzy totally ordered set or a fuzzy chain.

Definition 2 ([8]): Let $(\mathrm{R}, \vee, \wedge, 0)$ be an algebra of type $(2,2,0)$ and $(\mathrm{R}, \mathrm{A})$ be a fuzzy poset.Then we call $L=(R, A)$ is an Almost Distributive Fuzzy Lattice (ADFL) if the following axioms are satisfied:

1) $A(a, a \vee 0)=A(a \vee 0, a)=1$;

2) $A(0,0 \wedge a)=A(0 \wedge a, 0)=1$;

3) $A((a \vee b) \wedge c,(a \wedge c) \vee(b \wedge c))=A((a \wedge c) \vee(b \wedge c),(a \vee$ b) $\wedge c)=1$; 
4) $A(a \wedge(b \vee c),(a \wedge b) \vee(a \wedge c))=A((a \wedge b) \vee(a \wedge c), a \wedge$ $(b \vee c))=1$

5) $A(a \vee(b \wedge c),(a \vee b) \wedge(a \vee c))=A((a \vee b) \wedge(a \vee c), a \vee$ $(b \wedge c))=1$

6) $A((a \vee b) \wedge b, b)=A(b,(a \vee b) \wedge b)=1$; for all $a, b, c \in R$.

Definition 3 ([8]): Let L be an ADFL . Then for any $a, b$ $\in \mathrm{R}, \mathrm{a} \leq \mathrm{b}$ if and only if $\mathrm{A}(\mathrm{a}, \mathrm{b})>0$.

As a result of this definition, for any $a, b \in R, A(a, b)>0$ if and only if $a \wedge b=a$ or $a \vee b=b$, and hence we have the following Theorem which are the main results of [8].

Theorem 4 ([8]): Let $L=(R, A)$ be an ADFL. Then for each $\mathrm{a}, \mathrm{b}$ and $\mathrm{c}$ in $\mathrm{R}$ :

1) If $A(a, b)>0$ then $A(b, a)=0$, where $a \neq b$;

2) $A(a \wedge b, b)>0$ and $A(b \wedge a, a)>0$;

3) $\mathrm{A}(\mathrm{a}, \mathrm{a} \vee \mathrm{b})>0$ and $A(b, b \vee a)>0$;

4) $A(a \wedge b, b \wedge a)>0$ and $A(b \wedge a, a \wedge b)>0$ whenever $A(a, b)>0$

5) $A(a \vee b, b \vee a)>0$ and $A(b \vee a, a \vee b)>0$, whenever $A(a, b)>0$

6) $A(0, a \wedge 0)>0$ and $A(a, 0 \vee a)>0$;

7) $A(a, a \wedge a)>0$ and $A(a \vee a, a)>0$

8) $A((a \wedge b) \vee b, b)=1$ and $A(a \vee(a \wedge b), a)=1$

9) $A(a, a \wedge(a \vee b))=1$ and $A(a,(a \vee b) \wedge a)=1$

10) $A(a \vee(a \wedge b), a \wedge(a \vee b))=1$;

11) $A(a \vee(b \wedge a),(a \vee b) \wedge a)=1$

12) $A(a \vee b, a)>0$ if and only if $A(b, a \wedge b)>0$;

13) $A(a \vee b, b)>0$ and $A(b, a \vee b)>0$ iff $A(a \wedge b, a)>0$ and $A(a, a \wedge b)>0$

14) $A((a \vee b) \wedge c,(b \vee a) \wedge c)>0$ and $A((b \vee a) \wedge c,(a \vee b) \wedge$ c) $>0$

15) $A(a \wedge(b \wedge c),(a \wedge b) \wedge c)=1$;

16) $A(a \wedge b \wedge c, b \wedge a \wedge c)>0$ and $A(b \wedge a \wedge c, a \wedge b \wedge c)>0$.

Definition 5 ([9]): Let $L$ be an $A D F L$ and let $I$ be any non empty subset of $R$. $I$ is said to be an ideal of an ADFL $L$, if it satisfies the following axioms:

(i) $a, b \in I$ implies that $a \vee b \in I$

(ii) $a \in I$ and $b \in R$ implies that $a \wedge b \in I$.

Definition 6 ([9]): Let $L$ be an $A D F L$ and let $F$ be any non empty subset of $R$. Then $F$ is said to be filters of an ADFL $L$, if it satisfies the following axioms:

(i) $a, b \in F$ implies that $a \wedge b \in F$

(ii) $a \in F$ and $b \in R$ implies that $a \vee b \in F$.

Theorem 7 ([9]): Let $L$ be an ADFL and $S$ be any non empty subset of R. Then, $(S]_{A}=\left\{x \in R / A\left(x,\left(\bigvee s_{i}\right)_{i=1}^{n} \wedge x\right)>0\right.$, where $s_{i} \in S$ and $\left.n \in Z^{+}\right\}$is the smallest ideal of $L$ containing $S$.

Theorem 8 ([9]): Let $L$ be an ADFL and $\mathrm{F}$ be any non empty subset of R. Then $[F)_{A}=\left\{x \in R / A\left(\bigwedge_{i=1}^{n} f_{i}, x \wedge\left(\bigwedge_{i=1}^{n} f_{i}\right)\right)>\right.$ 0 , for some $f_{i} \in F$ and $\left.n \in Z^{+}\right\}$is the smallest filter of $L$ containing $\mathrm{F}$.

Theorem 9 ([9]): Let $I$ and $J$ are two ideals of an ADFL $L$. Then $I \wedge J$ and $I \bigvee J$ are also ideals of L, where $I \wedge J=I \cap J$ and $I \bigvee J=\{x \vee y / x \in I$ and $y \in J\}$.

Theorem 10 ([9]): Let $F$ and $G$ are two filters of an ADFL $L=(R, A)$. Then $F \wedge G$ and $F \bigvee G$ are also filters of $\mathrm{L}$, where $F \wedge G=F \cap G$ and $F \bigvee G=\{x \wedge y / x \in F$ and $y \in G\}$.

Definition 11 ([10]): Let $L$ be an $A D F L$ and let $a \in R$. An ideal generated by a singleton set $\{a\}$ denoted by $(a]_{A}$ is a principal ideal of L. Similarly, A filter generated by a singleton set $\{a\}$ denoted by $[a)_{A}$ is a principal filter of $\mathrm{L}$ generated by $a$.

Lemma 12 ([10]): Let $L$ be an $A D F L$ and let $a \in R$. The set $\{x \in R / A(x, a \wedge x)>0\}$ and $\{x \in R / A(a, x \wedge a)>0\}$ are principal ideal and principal filter of $\mathrm{L}$ generated by $a$, respectively.

Corollary 13 ([10]): Let $L$ be an $A D F L$ and let $a \in R$. Then for any element $a$ of $R, a \in(a]_{A}$ and $a \in[a)_{A}$.

Corollary 14 ([10]): Let $L$ be an $A D F L$. For any $a, b \in R$,

(i) $(a]_{A} \vee(b]_{A}=(a \vee b]_{A}=(b \vee a]_{A}$,

(ii) $(a]_{A} \wedge(b]_{A}=(a \wedge b]_{A}=(b \wedge a]_{A}$,

(iii) $[a)_{A} \bigvee[b)_{A}=[a \wedge b)_{A}=[b \wedge a)_{A}$,

(iv) $[a)_{A} \wedge[b)_{A}=[a \vee b)_{A}=[b \vee a)_{A}$.

Theorem 15 ([10]): Let $L$ be an $A D F L$. Then for any $a, b \in$ $R$, the followings are equivalent:

(i) $(a]_{A} \subset(b]_{A}$

(ii) $A(x, b \wedge x)>0$, for all $x \in(a]_{A}$

(iii) $A(b \vee x, b)>0$, for all $x \in(a]_{A}$

(iv) $[b)_{A} \subset[a)_{A}$

\section{THE FUZZY LATTICE OF IDEALS AND FILTERS OF AN Almost Distributive FuzZy LATtice}

In this section we introduce the concept of a fuzzy lattice by defining a fuzzy relation $\mathrm{B}$ on the set containing all ideals $I_{A}(L)$, and on the set containing all filters $F_{A}(L)$ of a given Almost Distributive Fuzzy Lattice L. In addition, we proved that a fuzzy poset $\left(P I_{A}(L), B\right)$ and $\left(P F_{A}(L), B\right)$ forms a fuzzy distributive lattice, where $P I_{A}(L)$ is the set containing all principal ideals, and $P F_{A}(L)$ denotes the set containing all filters of a given ADFL L respectively. Moreover in this section, we introduce ideals and filters of the two fuzzy distributive lattice $\left(P I_{A}(L), B\right)$ and $\left(P F_{A}(L), B\right)$ induced by arbitrary ideal I and filter $F$ of an ADFL L.

In this paper, $I_{A}(L)$ and $F_{A}(L)$ represents, the set containing all ideals and the set containing all filters of an ADFL L respectively.

Definition 16 ([7]): Let (X, A) be a fuzzy poset and let $B \subset X$. An element $u \in X$ is said to be an upper bound for a subset $\mathrm{Y}$ if and only if $A(b, u)>0$ for all $b \in Y$. An upper bound $u_{0}$ for $\mathrm{Y}$ is the least upper bound of $\mathrm{Y}$ if and only if $A\left(u_{0}, u\right)>0$ for every upper bound $\mathrm{u}$ of $\mathrm{Y}$. An element $v \in X$ is said to be a lower bound for a subset $\mathrm{Y}$ if and only if $A(v, b)>0$ for all $b \in B$. A lower bound $v_{0}$ for $\mathrm{Y}$ is the greatest lower bound of $\mathrm{Y}$ if and only if $A(v, v 0)>0$ for every lower bound $\mathrm{v}$ for $\mathrm{Y}$.

Consider a fuzzy relation $B$ on $I_{A}(L)$, and we have the following proposition.

Proposition 17: Let L be an ADFL and $\left(I_{A}(L), R\right)$ be a fuzzy Poset. For any $I, J \in I_{A}(L), B(I, J)>0$ if and only if for all $i \in I$ there exists $j \in J$ such that $A(i, j)>0$.

Proof: Let $I, J \in I_{A}(L)$. Suppose $B(I, J)>0$, and let $i \in I$. Since $I \subset J, i \in J$. Then $A(i, i)>0$.

Conversely, suppose for all $i \in I$ there exists $j \in J$ such that $A(i, j)>0$. Since $\mathrm{J}$ is an ideal, $i \in J$. Hence $B(I, J)>0$.

Proposition 18: Let L be an ADFL and $\left(F_{A}(L), R\right)$ be a fuzzy Poset. For any $F, G \in F_{A}(L), B(F, G)>0$ if and only if for all $f \in F$ there exists $g \in G$ such that $A(g, f)>0$. 
Proof: Let $F, G \in F_{A}(L)$. Suppose $B(F, G)>0$, and let $f \in F$. Since $F \subset G, f \in G$. Then $A(f, f)>0$.

Conversely, suppose for all $f \in F$ there exists $g \in G$ such that $A(g, f)>0$. Since $\mathrm{G}$ is an filter, $f \in G$. Hence $B(F, G)>0$.

Definition 19 ([7]): Let $(R, A)$ be a fuzzy poset. $(R, A)$ is a fuzzy lattice if and only if $x \vee y$ and $x \wedge y$ exist for all $x, y \in R$.

Definition 20 ([7]): Let (R, A) be a fuzzy lattice. (R, A) is distributive if and only if $x \wedge(y \vee z)=(x \wedge y) \vee(x \wedge z)$ and $(x \vee y) \wedge(x \vee z)=x \vee(y \wedge z)$ for all $x, y, z \in R$.

Theorem 21: Let L be an ADFL and $\left(I_{A}(L), B\right)$ be a fuzzy poset. Then $\left(I_{A}(L), B\right)$ forms a fuzzy lattice.

Proof: Let $I$ and $J$ are any arbitrary elements of $I_{A}(L)$. By Theorem 9, both $I \vee J$ and $I \wedge J$ exist and they are in $\left(I_{A}(L)\right.$. First we show that $I \vee J$ and $I \wedge J$ are an upper bound and lower bound of the set containing $\mathrm{I}$ and $\mathrm{J}$ respectively.

Let $i \in I$. Then $i=i \vee 0 \in I \vee J$. Hence $B(I, I \vee J)>0$, and similarly $B(J, I \vee J)>0$. Thus $I \bigvee J$ is the upper bound of the set containing $\mathrm{I}$ an $\mathrm{J}$.

Let $i \in I \wedge J$, Then $i \in I$ and $i \in J$. Hence $B(I \wedge J, I)>0$ and $B(I \wedge J, J)>0$. Thus $I \wedge J$ is the lower bound of $I$ and $J$.

Assume there exist an ideal $\mathrm{K}$ of $\mathrm{L}$ such that $B(I, K)>0$ and $(J, K)>0$.

Let $x \in I \bigvee J$. Then there exists $i \in I$ and $j \in J$ such that $x=$ $i \vee j$. Since $B(I, K)>0$ and $B(J, K)>0$, there exist $k_{1}, k_{2} \in K$ such that $A\left(i, k_{1}\right)>0$ and $A\left(j, k_{2}\right)>0$, and it implies that $i, j \in K$. Hence $x=i \vee j$, and then $B(I \bigvee J, K)>0$. Thus $I \bigvee J$ is the smallest upper bound of I and $\mathrm{J}$.

Again, assume there exists an ideal $\mathrm{N}$ of $\mathrm{L}$ such that $B(N, I)>$ 0 and $B(N, J)>0$. Let $n \in N$, then there exists $i \in I$ and $j \in J$ such that $A(n, i)>0$ and $A(n, j)>0$, and it implies that $n \in I$ and $n \in J$. Hence $n \in I \wedge J$ and then $B(N, I \wedge J)>0$. Thus $I \wedge J$ is the greatest lower bound of I and $\mathrm{J}$.

Therefore, $\left(I_{A}(L), B\right)$ forms a fuzzy lattice, and we call it a fuzzy lattice of all ideals of an ADFL L.

Theorem 22: Let L be an ADFL and $\left(F_{A}(L), B\right)$ be a fuzzy poset. Then $\left(F_{A}(L), B\right)$ forms a fuzzy lattice.

Proof: Let $F$ and $G$ are any arbitrary elements of $F_{A}(L)$. By Theorem 10, $F \vee G$ and $F \wedge G$ both exist and they are in $\left(F_{A}(L)\right.$. First we show that $F \vee G$ and $F \wedge G$ are an upper bound and lower bound of the set containing $\mathrm{F}$ and $\mathrm{G}$ respectively.

To show $F \vee G$ is the upper bound of $\mathrm{F}$ and $\mathrm{G}$, let $f \in F$. Then $f \vee g \in G$, where $g \in G$ and it implies that $f \wedge(f \vee g) \in F \vee G$. Then $A(f \wedge(f \vee g), f)>0$ and then $B(F, F \vee G)>0$

Again let $g \in G$, then $f \vee g \in F$, where $f \in F$, and it implies $(f \vee g) \wedge g \in F \vee G$. Hence, $A((f \vee g) \wedge g, g)>0$, for every $g \in G$ and then $B(G, F \bigvee G)>0$.

Thus $F \bigvee G$ is an upper bound of $\mathrm{F}$ and $\mathrm{G}$.

Similarly, to show $F \wedge G$ is the lower bound of $\mathrm{F}$ and $\mathrm{G}$, let $x \in F \wedge G$. Then there exists $f \in F$ and $g \in G$ such that $x=$ $f \vee g$. Since $\mathrm{F}$ and $\mathrm{G}$ are filters of $\mathrm{L}, x \in F$ and $x \in G$. Hence, $B(F \wedge G, F)>0$ and $B(F \wedge G, G)>0$. Thus $F \wedge G$ is the lower bound of $\mathrm{F}$ and $\mathrm{G}$.

To show $F \wedge G$ is the list upper bound of $\mathrm{F}$ and $\mathrm{G}$, let us assume there exists a filter $\mathrm{H}$ of $\mathrm{L}$ such that $B(F, H)>0$ and $B(G, H)>0$. Let $a \in F \bigvee G$. Then there exists $\mathrm{f}$ and $\mathrm{g}$ in $\mathrm{F}$ and $\mathrm{G}$ respectively, such that $a=f \wedge g$. Since $B(F, H)>0$ and $B(G, H)>0$, there exists $h_{1}$ and $h_{2}$ in $\mathrm{H}$ such that $A\left(h_{1}, f\right)>0$ and $A\left(h_{2}, g\right)>0$, and then $\mathrm{f}$ and $\mathrm{g}$ belongs to $\mathrm{H}$ and $\mathrm{H}$, and it follows that $f \wedge g \in H$. Hence, $B(F \bigvee G, H)>0$ and this follows from the fact that $(f \wedge g, a)>0$. Thus $F \bigvee G$ is the least upper bound of $\mathrm{F}$ and $\mathrm{G}$.

Finally, assume there exists a filter $\mathrm{M}$ of an ADFL L such that $B(M, F)>0$ and $B(M, G)>0$, and let $m \in M$. Then there exists $\mathrm{f}$ and $\mathrm{g}$ in $\mathrm{F}$ and $\mathrm{G}$ respectively such that $A(f, m)>0$ and $A(g, m)>0$. Then $A(f \vee g, m)=A((f \vee g) \wedge m, m)>0$, and it implies that $B(M, F \wedge G)>0$. Hence, $F \wedge G$ is the greatest lower bound of $\mathrm{F}$ and $\mathrm{G}$.

Therefore, $\left(F_{A}(L), B\right)$ forms a fuzzy lattice, and we call it the fuzzy lattice of all filters of an ADFL L.

From Theorem 9 and Theorem 10, we already have seen that, in a given ADFL L if $\left(I_{A}(L), B\right)$ and $\left(F_{A}(L), B\right)$ are fuzzy posets, then both forms a fuzzy lattice, and we call them a fuzzy lattice of all ideals and the fuzzy lattice of all filters of an ADFL L respectively. Now lets consider the set of all principal ideals $P I_{A}(L)$ of an ADFL L, and the set of all principal filters $P F_{A}(L)$ of an ADFL L. Clearly $P I_{A}(L)$ and $P F_{A}(L)$ are subset of $I_{A}(L)$ and $F_{A}(L)$ respectively. Then as an immediate consequence of the above two Theorems, we have the following Corollaries.

Corollary 23: Let $P I_{A}(L)$ be the set of all principal ideals of an ADFL L, and $\left(P I_{A}(L), B\right)$ be a fuzzy poset. Then $\left(P I_{A}(L), B\right)$ forms a distributive fuzzy lattice.

Proof: The proof is direct from Theorem 21 and Corollary 14 (i) and (ii).

Corollary 24: Let $P F_{A}(L)$ be the set of all principal filters of an ADFL L, and $\left(P F_{A}(L), B\right)$ be a fuzzy poset. Then $\left(P F_{A}(L), B\right)$ forms a distributive fuzzy lattice.

Proof: The proof is direct from Theorem 22 and Corollary 14 (iii) and (iv).

Definition 25 ([11]): Let $(H, C)$ be a fuzzy lattice and I be any non empty subset of $\mathrm{H}$. I is an ideal of $(H, C)$, if the following two conditions hold true.

(i) $i_{1}, i_{2} \in I$ implies $i_{1} \vee i_{2} \in I$

(ii) $i \in I$ and $x \in H$ such that $C(x, i)>0$ implies $x \in I$

Definition 26 ([11]): Let $(H, C)$ be a fuzzy lattice and $\mathrm{F}$ be any non empty subset of $\mathrm{H}$. F is an ideal of $(H, C)$, if the following two conditions hold true.

(i) $f_{1}, f_{2} \in F$ implies $f_{1} \wedge f_{2} \in F$

(ii) $f \in F$ and $x \in H$ such that $C(f, x)>0$ implies $x \in F$ From Corollary 23 and 28 we have seen that, $\left(P I_{A}(L), B\right)$ and $\left(P F_{A}(L), B\right)$ forms a fuzzy lattice, whenever both are fuzzy posets, and in the following two Lemmas, we define an ideal and filters of a fuzzy lattice $\left(P I_{A}(L), B\right)$ and $\left(P F_{A}(L), B\right)$ which are induced by an ideal I and a filter F of an ADFL L.

We first start by ideals and filters of a fuzzy lattice $\left(P I_{A}(L), B\right)$, and in this paper $L_{B}^{\downarrow}$ denotes a distributive fuzzy lattice $\left(P I_{A}(L), B\right)$ and $L_{B}^{\uparrow}$ denotes a distributive fuzzy lattice $\left(P F_{A}(L), B\right)$. Consider two non empty subset I and $\mathrm{F}$ of $\mathrm{R}$. Define $I_{A}^{\downarrow}, I_{A}^{\uparrow}, F_{A}^{\downarrow}$, and $F_{A}^{\uparrow}$ by:

$I_{A}^{\downarrow}=\left\{(i]_{A} / i \in I\right\}, I_{A}^{\uparrow}=\left\{[i)_{A} / i \in I\right\}, F_{A}^{\downarrow}=\left\{(f]_{A} / f \in F\right\}$, and $F_{A}^{\uparrow}=\left\{[f)_{A} / f \in F\right\}$. It is clear that $I_{A}^{\downarrow}$ and $F_{A}^{\downarrow}$ are subsets of $P I_{A}(L)$ and $I_{A}^{\uparrow}$ and $F_{A}^{\uparrow}$ are subsets of $P F_{A}(L)$. Then we have the following Theorem.

Theorem 27: Let I be any non empty subset of $\mathrm{R}$, and $\mathrm{L}$ be an ADFL. Then the followings are equivalent: 
1) I is an ideal of $\mathrm{L}$;

2) $I_{A}^{\downarrow}$ is an ideal of a distributive fuzzy lattice $L_{B}^{\downarrow}$;

3) $I_{A}^{\uparrow}$ is a filter of a distributive fuzzy lattice $L_{B}^{\uparrow}$.

Proof: (1) $\Rightarrow$ (2) Suppose I is an ideal of L. We need to show that $I_{A}^{\downarrow}$ is an ideal of $L_{B}^{\downarrow}$.

(a) Let $\left(i_{1}\right]_{A}$ and $\left(i_{2}\right]_{A}$ be any two arbitrary elements of $I_{A}^{\downarrow}$. Then $\left(i_{1}\right]_{A} \bigvee\left(i_{2}\right]_{A}=\left(i_{1} \vee i_{2}\right]_{A}$. Since $i_{1} \vee i_{2} \in I,\left(i_{1}\right]_{A} \bigvee\left(i_{2}\right]_{A}=$ $\left(i_{1} \vee i_{2}\right]_{A} \in I_{A}^{\downarrow}$.

(b) Let $(i]_{A} \in I_{A}^{\downarrow}$ and $(x]_{A} \in P I_{A}(L)$ such that $B\left((x]_{A},(i]_{A}\right)>0$. Then there exists $y \in(i]_{A}$ such that $A(x, y)>0$, in particular $A(x, i \wedge y)>0$. since $\mathrm{I}$ is an ideal of $\mathrm{L}$ and $i \wedge y \in I, x \in I$, and then $(x]_{A} \in I_{A}^{\downarrow}$. Therefore, $I_{A}^{\downarrow}$ is an ideal of $L_{B}^{\downarrow}$.

(2) $\Rightarrow$ (3) Suppose $I_{A}^{\downarrow}$ is an ideal of $L_{B}^{\downarrow}$. We need to show that $I_{A}^{\uparrow}$ is a filter of $L_{B}^{\uparrow}$.

(a) Let $\left[i_{1}\right)_{A}$ and $\left[i_{2}\right)_{A}$ be any two arbitrary elements of $I_{A}^{\uparrow}$. Then $\left[i_{1}\right)_{A} \wedge\left(i_{2}\right)_{A}=\left[i_{1} \vee i_{2}\right)_{A}$. Since $\mathrm{I}$ is an ideal of $\mathrm{L}, i_{1} \vee$ $i_{2} \in I$ and it follows that $\left[i_{1} \vee i_{2}\right)_{A} \in I_{A}^{\uparrow}$. (b) Let $[i) \in I_{A}^{\uparrow}$ and $[x)_{A} \in P F_{A}(L)$ such that $B\left([i)_{A},[x)_{A}\right)>0$. Then $A(x, i \wedge x)>0$ or $A(i \vee x, i)>0$. Since $\mathrm{I}$ is an ideal of $\mathrm{L}, x \in I$. Hence $(x]_{A} \in I_{A}^{\uparrow}$. Thus $I_{A}^{\uparrow}$ is a filter of $L_{B}^{\uparrow}$.

(3) $\Rightarrow$ (1) Suppose $I_{A}^{\uparrow}$ is a filter of a distributive fuzzy lattice $L_{B}^{\uparrow}$. We need to show that $\mathrm{I}$ is an ideal of $\mathrm{L}$.

(a) Let $i_{1}, i_{2} \in I$. Then $\left[i_{1}\right)_{A},\left[i_{2}\right)_{A} \in I_{A}^{\uparrow}$ and since $I_{A}^{\uparrow}$ is a filter of $L_{B}^{\uparrow},\left[i_{1}\right)_{A} \wedge\left(i_{2}\right)_{A}=\left[i_{1} \vee i_{2}\right)_{A} \in I_{A}^{\uparrow}$. Hence $i_{1} \vee i_{2} \in I$.

(b) Let $i \in I, x \in R$. Hence $[i)_{A} \in I_{A}^{\uparrow}$. Since $B\left([i)_{A},[i)_{A} \vee[x)_{A}\right)>$ 0 and $I_{A}^{\uparrow}$ is a filter of $L_{B}^{f},[x \wedge i)_{A}=[i \wedge x)_{A}=[i)_{A} \vee[x)_{A} \in I_{A}^{\uparrow}$. Hence, $x \wedge i \in I$. Therefore, $\mathrm{I}$ is an ideal of $\mathrm{L}$.

Theorem 28: Let $\mathrm{F}$ are be any non empty subset of $\mathrm{R}$, and $\mathrm{L}$ be an ADFL. Then the followings are equivalent.

1) $F$ is a filter of $L$;

2) $F_{A}^{\downarrow}$ is a filter of a distributive fuzzy lattice $L_{B}^{\downarrow}$;

3) $F_{A}^{\uparrow}$ is an ideal of a distributive fuzzy lattice $L_{B}^{\uparrow}$.

Proof: $(1) \Rightarrow(2)$ Suppose $\mathrm{F}$ is a filter of $\mathrm{L}$. Then we need to show that $F_{A}^{\downarrow}$ is a filter of $L_{B}^{\downarrow}$.

(a) Let $\left(f_{1}\right]_{A}$ and $\left(f_{2}\right]_{A}$ are any two arbitrary elements of $F_{A}^{\downarrow}$. Then $\left(f_{1}\right]_{A} \wedge\left(f_{2}\right]_{A}=\left(f_{1} \wedge f_{2}\right]_{A} \in F_{A}^{\downarrow}$, since $\mathrm{F}$ is a filter and $f_{1} \wedge f_{2} \in F$.

(b) Let $(f]_{A} \in F_{A}^{\downarrow}$ and $(x]_{A} \in P I_{A}(L)$ such that $B\left((i]_{A},(x]_{A}\right)>0$. Then $f \in(x]_{A}$ and it implies that $A(f, x \wedge f)>0$ or $A(x \vee f, x)>0$. Hence, $x \in F$ and thus $(x]_{A} \in F_{A}^{\downarrow}$. Therefore, $F_{A}^{\downarrow}$ is a filter of a distributive fuzzy lattice $L_{B}^{\downarrow}$.

(2) $\Rightarrow$ (3) Suppose $F_{A}^{\downarrow}$ is a filter of $L_{B}^{\downarrow}$. Then we need to show that $F_{A}^{\uparrow}$ is an ideal of $L_{B}^{\uparrow}$.

(a) Let $\left[f_{1}\right)_{A}$ and $\left[f_{2}\right)_{A}$ are any two arbitrary elements of $F_{A}^{\uparrow}$. Then $\left[f_{1}\right)_{A} \bigvee\left[f_{2}\right)_{A}=\left[f_{1} \wedge f_{2}\right)_{A} \in F_{A}^{\uparrow}$, since $\mathrm{F}$ is a filter and $f_{1} \wedge f_{2} \in F$.

(b) Let $[f)_{A} \in F_{A}^{\uparrow}$ and $[x)_{A} \in P F_{A}(L)$ such that $B\left([x)_{A},[f)_{A}\right)>0$. Then $x \in[f)_{A}$ and it implies that $A(f, x \wedge f)>0$ or $A(x \vee f, x)>0$. Since $f \in F$ and $\mathrm{F}$ is a filter, $x \in F$ and it implies that $[x)_{A} \in F_{A}^{f}$. Therefore, $F_{A}^{\uparrow}$ is an ideal of a distributive fuzzy lattice $L_{B}^{f}$.

(3) $\Rightarrow(1)$ Suppose $F_{A}^{\uparrow}$ is an ideal of a distributive fuzzy lattice $L_{B}^{\uparrow}$. We need to show that $\mathrm{F}$ is a filter of $\mathrm{L}$.

(a) Let $f_{1}, f_{2} \in F$. Then $\left[f_{1}\right)_{A},\left[f_{2}\right)_{A} \in F_{A}^{\uparrow}$. Since $F_{A}^{\uparrow}$ is an ideal of $L_{B}^{\uparrow},\left[f_{1}\right)_{A} \bigvee\left[f_{2}\right)_{A}=\left[f_{1} \wedge f_{2}\right)_{A} \in F_{A}^{\uparrow}$. Hence $f_{1} \wedge f_{2} \in F$. (b) Let $f \in F, \quad y \in R$. Hence $[f)_{A} \in F_{A}^{\uparrow}$. Since $B\left([x)_{A} \wedge[i)_{A},[i)_{A}\right)>0$ and $F_{A}^{\uparrow}$ is an ideal of $L_{B}^{f}$, $[i \vee x)_{A}=[x \vee i)_{A}=[x)_{A} \wedge[i)_{A} \in F_{A}^{\uparrow}$. Hence, $i \wedge x \in F$. Therefore, $\mathrm{F}$ is a filter of $\mathrm{L}$.

\section{REFERENCES}

[1] U. Swamy and G. Rao, "Almost distributive lattices," Journal of the Australian Mathematical Society (Series A), vol. 31, no. 01, pp. 77-91, 1981.

[2] L. Zadeh, "Fuzzy sets," Information and control, vol. 8, no. 3, pp. 338353, 1965.

[3] E. Sanchez, "Resolution of composite fuzzy relation equations," Information and control, vol. 30, no. 1, pp. 38-48, 1976.

[4] J. Goguen, "L-fuzzy sets," Journal of mathematical analysis and applications, vol. 18, no. 1, pp. 145-174, 1967.

[5] Y. Bo and W. Wangming, "Fuzzy ideals on a distributive lattice," Fuzzy sets and systems, vol. 35, no. 2, pp. 231-240, 1990.

[6] N. Ajmal and K. Thomas, "Fuzzy lattices," Information sciences, vol. 79 , no. 3-4, pp. 271-291, 1994.

[7] I. Chon, "Fuzzy partial order relations and fuzzy lattices," Korean $J$ Math, vol. 17, no. 4, pp. 361-374, 2009.

[8] A. Berhanu, G. Yohanes, and T. Bekalu, "Almost distributive fuzzy lattice," To be communicated.

[9] A. Berhanu and T. Bekalu, "Ideals and filters of an almost distributive fuzzy lattice," To be communicated, 2016.

[10] - "Principal ideals and principal filters of an almost distributive fuzzy lattice," To be communicated, 2016.

[11] I. Mezzomo, B. Bedregal, and R. Santiago, "On fuzzy ideals of fuzzy lattice," in IEEE International Conference on Fuzzy Systems (FUZZIEEE), 2012, pp. 1-5. 